\title{
Monochromatic and Polychromatic Serial Crystallography at the Advanced Photon Source
}

R.F. Fischetti ${ }^{1}$, J. Martin-Garcia ${ }^{2,2}$, N. Zatsepin ${ }^{2,4}$, N. Stander ${ }^{2,4}$, L. Zhu ${ }^{2,3}$, G. Subramanian $^{2,4}$, G. Nelson ${ }^{2,4}$, J. Coe ${ }^{2,3}$ N. Nagaratnam ${ }^{2,3}$, S. Roy-Chowdhury ${ }^{2,3}$, D. Kissick $^{1}$, A. Ishchenko ${ }^{5}$, C. Conrad ${ }^{2,3}$, G. Ketawala ${ }^{2,3}$, D. James $^{6}$, J. Zook ${ }^{2,3}$, C. Ogata ${ }^{1}$, N. Venugopalan ${ }^{1}$, S. $\mathrm{Xu}^{1}$, A. Meents ${ }^{7}$, V. Srajer ${ }^{8}$, R. Henning ${ }^{8}$, H. Chapman ${ }^{7}$, J. Spence $^{2,4}$, U. Weierstall ${ }^{2,4}$, V. Cherezov', P. Fromme ${ }^{2,3}$, W. Liu ${ }^{2,3}$

${ }^{1}$ Advanced Photon Source, Argonne National Laboratory, Chicago, IL, USA

${ }^{2}$ Center for Applied Structural Discovery, Biodesign Institute, Arizona State University, Tempe, AZ, USA

${ }^{3}$ School of Molecular Sciences, Arizona State University, Tempe, AZ, USA

${ }^{4}$ Department of Physics, Arizona State University, Tempe, AZ, USA

${ }^{5}$ Department of Chemistry, University of Southern California, Los Angeles, CA, USA

${ }^{6}$ Paul Scherrer Institute, Villigen, Switzerland

${ }^{7}$ Centre for Free-Electron Laser Science, Deutsches Elektronen-Synchrotron, Hamburg, Germany

${ }^{8}$ BioCARS, University of Chicago, IL, USA

Serial femtosecond crystallography (SFX) is limited by scarcity of XFEL facilities while synchrotron sources are becoming viable options as alternatives for serial millisecond crystallography (SMX) with monochromatic and serial picosecond crystallography with polychromatic or pink-beam. Here, we present injector-based SMX experiments carried out at the Advanced Photon Source (APS). Monochromatic beam experiments were conducted at the GM/CA 23-ID-D beamline. Micro-crystals of a wide variety of proteins including lysozyme, thaumatin, PSII, phycocyanin, human adenosine $A_{2 A}$ receptor $\left(A_{2 A} A R\right)$, beta-2 adrenergic receptor $\left(\beta_{2} A R\right)$, KDO8PS, FLPP3, and proteinase $K$ were screened. Crystals were delivered to the beam suspended in lipidic cubic phase, agarose or a high molecular weight PEO ( $M W=8,000,000)$, using a LCP injector. For each protein target, tens to hundreds of thousands of diffraction patterns were collected by a Pilatus $6 \mathrm{M}$ detector in shutterless mode at a repetition rate of $10 \mathrm{~Hz}$, using a photon energy of $12 \mathrm{keV}$ and $10 \mu \mathrm{m}$ diameter beam size. Inhouse hit-finding software developed at APS and SFX data-reduction and analysis software suites, Cheetah and CrystFEL, enabled efficient SMX data monitoring, reduction and processing. From these experiments the structures of the $A_{2 A} A R$, phycocyanin, FLPP3, proteinase $K$ and lysozyme were determined to $3.1 \AA$, $3.1 \AA$, $3.0 \AA, 2.65 \AA$, and $2.05 \AA$ resolution, respectively. Pink beam experiments were conducted at the BioCARS beamline (14-ID-B) on micro-crystals in which each crystal was exposed to the X-rays from only a single $\sim 100$ picosecond bunch or 4 consecutive bunches separated by 153 ns for a total exposure time of 460 ns. The broad bandwidth (5\%) yielded full reflections. Thus only a few thousand diffraction patterns were recorded to build a complete dataset of phycocyanin to $2.7 \AA$ resolution, and over 60,000 images were collected from proteinases $K$ with rates varying from $3.2 \%-6.3 \%$. The structure of proteinase $\mathrm{K}$ was determined to $1.8 \mathrm{~A}$ resolution. Preliminary experiments were carried out with the high viscosity injector for $A_{2 A} A R$, lysozyme, and phycocyanin. In order to collect complete data sets more data will be collected for these four proteins at an upcoming beamtime. Monochromatic and pink beam experiments demonstrate the feasibility of serial data collection at the APS using micro-crystals. Upcoming developments in beamline optics will 
increase intensity by a factor of ten. In addition, the intensity will also be increased by another factor of ten from the planned APS-U upgrade enabled by the two orders of magnitude increase in Brightness. All these developments will enable the use of smaller micro-crystals as well as the SMX of larger macromolecules. 